\title{
ARTICLE
}

\section{Compassion-focused therapy with children and adolescents}

\author{
Carlos Carona, Daniel Rijo, Céu Salvador, Paula Castilho \& Paul Gilbert
}

Carlos Carona is a clinical psychologist with the Cerebral Palsy Association of Coimbra, Portugal, and a researcher in the CognitiveBehavioural Center for Research and Intervention, University of Coimbra, and the Portuguese Federation of Cerebral Palsy Associations. He also delivers postgraduate cognitivebehavioural therapy (CBT) training. His interests include third-wave CBT, developmental psychopathology and paediatric psychology. Daniel Rijo is an assistant professor at the University of Coimbra and a researcher in the CognitiveBehavioral Center for Research and Intervention. His research focuses on personality disorders, antisocial behaviour, and the possibility of changing psychopathic traits in young offenders using a compassion-focused approach.

Céu Salvador is an assistant professor at the University of Coimbra and a researcher in the Cognitive-Behavioral Center for Research and Intervention. She has wide experience in delivering CBT for children and adolescents. Her research interests include CBT models and interventions for adolescents and adults. Paula Castilho is an assistant professor at the University of Coimbra. As well as her extensive clinical practice, she conducts research at the CognitiveBehavioral Center for Research and Intervention into evolutionary psychopathology and compassionfocused therapy. Paul Gilbert is Professor of Clinical Psychology at the University of Derby, UK. A Fellow of the British Psychological Society, he is an active researcher in shame-related processes in mood disorders and compassion as a focus for therapeutic interventions. Correspondence Dr Carlos Carona, University of Coimbra, Faculty of Psychology and Education Sciences, Cognitive and Behavioural Center for Research and Intervention, Rua do Colégio Novo, 3000-115 Coimbra, Portugal. Email: ccarona@fpce.uc.pt

\section{Copyright and usage}

(C) The Royal College of Psychiatrists 2017.

\begin{abstract}
SUMMARY
Compassion-focused therapy (CFT) is embedded in an evolutionary, functional analysis of psychopathology, with a focus on affiliative, caring and compassion processes. CFT has been applied in a number of adult settings, butits clinical applications in child and adolescent psychopathology and psychotherapy have not been systematically explored. This article describes the applications of CFT in paediatric populations. Specifically, the following developmental considerations are discussed: the unique importance of parent-child and attachment relationships for the development of self-compassion, being open to compassion from others and being compassionate to others; the potential effect of compassion training on the maturing brain (affective regulation systems); and the therapeutic targeting of shame and self-criticism to alleviate psychological distress and enhance the effectiveness of cognitivebehavioural interventions.
\end{abstract}

\section{LEARNING OBJECTIVES}

- Understand and differentiate the three affect regulation systems and their links to different forms of child and adolescent psychopathology

- Recognise the main components of compassionate mind training with children and adolescents, and related specific therapeutic strategies and exercises

- Acknowledge the importance of adopting a parent-child approach in CFT

\section{DECLARATION OF INTEREST}

None

During the past 20 years, there has been increasing research interest in the beneficial effects of cultivating compassion and prosocial behaviour. Effects have been demonstrated on a range of psychological processes (Keltner 2014), social relationships (Cozolino 2007; Crocker 2012), physiological processes (Klimecki 2014; Brown 2015) and genetic expression (Fredrickson 2013). Cultivating compassion for the self and others has also become a central focus for the development of psychotherapies (Gilbert 2010; Hoffmann 2011; Neff 2015). In this context, compassion-focused therapy (CFT) has emerged as one of the leading approaches, with an expanding evidence base (Leaviss 2015; Kirby 2016).

Compassion can be defined as sensitivity to suffering or distress in self and others, with a commitment to try to alleviate and prevent it (Gilbert 2013). Compassion sits within a family of prosocial motivations that also includes caring and altruism. In interpersonal relationships, compassion can be viewed as a flow: there is the compassion we feel for others, the compassion we are open to receive from others, and self-compassion. Each of these has a range of facilitators and inhibitors. For example, it is easier to be compassionate towards people we like than people we do not, easier to receive compassion from people we like, and easier to be self-compassionate about the things we like about ourselves rather than those aspects we do not like. The psychotherapeutic focus, therefore, is on understanding the facilitators and inhibitors of compassion in these different domains and working on their improvement.

\section{Attachment and interpersonal neurobiology}

Our understanding of prosocial motives and emotions, including compassion, has advanced through recent discoveries in several independent research fields, including developmental psychology and cognitive neuroscience. Particularly important is the understanding of neuroplasticity and neurogenesis, whereby social context plays an important part in choreographing and sculpting brain processes (Fredrickson 2013; Puglia 2015). Several studies in child development and neuroscience have helped therapists understand what happens to an individual's brain and subsequent psychological development when they experience various forms of early rearing difficulties (Siegel 2001; Gilbert 2014). Interactions with attachment figures are essential to creating contingent, attuned collaborative communication, to establish patterns by which the caregiver can regulate the child's positive and negative emotional states. These emotion-regulating interactions are required for the experientially influenced maturation of the infant's developing emotional and social brain (the ability to create a representation of the self and of the minds of 
others) (Schore 1994; Siegel 2001; Mikulincer 2007). Moreover, the quality of early relationships affects the development of internal working models of the self (e.g. as worthy or unworthy of care and support) and of others (e.g. as caring and available or threatening and unavailable) (Bowlby 1980; Baldwin 1997; Mikulincer 2007). Thus, these self-to-other scripts form the basis for subsequent self-to-self evaluations and determine predictions of one's own and others' behaviour in social interactions (Baldwin 1997).

\section{Early experiences and affect regulation systems}

Social interactions and the underlying emotion regulation processes are associated with specific neurohormones, as well as with a set of evolved central and peripheral physiological systems. These mechanisms can be conceptualised as integrating three different affect regulation systems, which work interactively and mutually affect each other (Depue 2005; Gilbert 2010):

- the threat defence system

- the resource-seeking system (the drive system)

- the soothing system (other terms for this include the contentment/affiliation and soothing system, the warmth/contentment system, and the soothing and social connectedness affect system).

Children who come from abusive, hostile, neglectful and/or threatening backgrounds are vulnerable to deficits in their affect regulation systems (Schore 1994). Feeling rejected, uncared for and unvalued is one of the most powerful elicitors of the stress response, and is related to physical and mental health problems (Cozolino 2007). Conversely, feeling cared for, supported and valued by others significantly influences physiological and emotional regulation, generating feelings of safeness and soothing (Depue 2005).

\section{The threat defence system}

The threat defence system is focused on the detection of threats and on the automatic activation of defensive emotions (e.g. anxiety, anger and disgust) and behaviours (e.g. fight, flight, submission and freezing). This system is linked to the secretion of the hormones serotonin and cortisol, and it operates through specific brain structures, such as the amygdala and the hypothalamic-pituitary-adrenal axis (LeDoux 2003; Depue 2005). The synaptic regulation of serotonin plays a part in the threat defence system - partly because of the defensive rule 'better safe than sorry' - which is an easily conditioned system and a source of psychopathology (LeDoux 2003). Negative early experiences overstimulate the threat defence system, which is designed to respond quickly and automatically to threat signals, while understimulating the soothing system (Gilbert 2014). The threat system is easily activated and can generate enduring negative affect and defensive behaviours (Perry 1995).

\section{The resource-seeking system}

The resource-seeking or drive system is mediated by the dopamine reward circuitry. This system is crucial to survival and prosperity, as it is responsible for the positive feelings (e.g. activation, pleasure, excitement and satisfaction) that guide and motivate us to seek out resources such as food.

\section{The soothing system}

The soothing system, characterised by positive feelings of warmth, soothing and well-being, is linked to endorphins and oxytocin and involves a state of quiescence, non-seeking and happiness/ contentment (Depue 2005). Maturation and activation of this system co-occur with the development of the attachment system, in which attachment figures are expected to act as reassuring and soothing agents (Gerhardt 2004). Thus, early attachment relationships will stimulate this system (e.g. through warmth and love signals), providing memories that will be available and recruited in the future, both in interpersonal contexts (e.g. forming affiliative bonds) and in selfregulation when facing stressful situations (Gilbert 2008a). Acting in a soothing and affiliative way with the self and others makes the world feel safer (decreasing stress and cortisol levels, shame feelings and behaviours, and negative evaluations about the self and others), promotes feelings of connectedness, deactivates the threat defence system, and provides tools and strategies to cope with adversity (Gilbert 2010).

\section{Activation of the threat defence system: shame and self-criticism}

Human beings are highly motivated to create positive images and positive affect in the minds of others and to be viewed as attractive social agents. Shame, as a self-conscious emotion, emerges from our evolved abilities to be aware of 'how we exist in the eyes of others', as a response to the social threat of being socially unattractive (Gilbert 2007). Shame has been defined as the experience of the self as unattractive, undesirable, worthless, inferior or defective in some way, and is linked to having flaws, failures and deficits exposed (Gilbert 2014). 
Gilbert's biopsychosocial approach posits two types of shame: external and internal shame (Gilbert 2007). External shame, in which the world is experienced as unsafe (e.g. others will be harsh and rejecting rather than supportive and forgiving), leads to the engagement in defensive manoeuvres, eliciting behavioural efforts to achieve a positive image in the mind of others (e.g. by submitting, appeasing or obeying). However, the internalisation of these experiences can result in devaluation of the self in line with those same negative experiences: the individual considers him- or herself to be flawed, inferior, defective and globally a failure (Gilbert 2010; Castilho 2014). These negative self-evaluations and feelings, known as self-criticism, can be seen as a form of self-to-self relationship, an internal process that triggers the same subordinated defeat strategies normally used to respond to external threat signals. These, when activated, result in negative emotions and psychopathology (Castilho 2015). Shame-proneness has been associated with increased vulnerabilities to psychopathology, both internalised (Tangney 2012; Matos 2013) and externalised (Gold 2011; Ribeiro da Silva 2015).

\section{Activation of the soothing system: self-compassion}

Feeling cared for, supported and understood helps us to understand our own minds and emotions and shapes our motives (Gilbert 2009). Several authors argue that feelings of safeness are central to the development of secure attachment bonds (Gerhardt 2004). Securely attached individuals seem to be more self-reassuring and warm towards themselves, as well as being more prosocial and prone to trust in others (Gilbert 2008b). Signals of compassion from others or from the self activate the soothing system, which is related to the caregiving mentality and associated with feelings of care, affiliation, soothing and safeness. Therefore, compassion involves several emotional, cognitive and motivational elements, including care for the welfare of others, sympathy, distress sensitivity, distress tolerance, empathy, non-judgement and the ability to create opportunities for growth and change with a warm attitude (Gilbert 2010).

An increasing number of studies have demonstrated an association between self-compassion (although measured in a different way to the model discussed here) and adaptive psychological functioning. Some studies have found selfcompassion to be negatively associated with depression and anxiety in adolescents and young adults, whereas others have suggested that, among college students, self-compassion is associated with adaptive coping and well-being in response to academic failure (for a review see Neff $\&$ Dahm, 2015). There is good evidence for the effectiveness of CFT in reducing many mental health problems (Kirby 2016).

\section{Case formulation in CFT}

\section{Developing motivating rationales}

The importance of a treatment rationale is well established in cognitive-behavioural therapies: the 'rationale' represents a common understanding between the therapist and the patient on the possible aetiologies of the difficulties experienced (why/how is this person having this problem?) and on the proposed intervention programme (what can we do to change it?). Moreover, the acceptance of a treatment rationale is associated with positive intervention outcomes (Addis 2000). The rationale can be viewed as a shared case formulation, and in CFT this formulation is regarded as a coconstructed process, aimed at enabling people to gain insight into how some of their basic defensive responses (such as anxiety and anger) may be quite automatic and trigger behavioural safety strategies such as withdrawal or submission (Gilbert 2013). There are four essential domains to look for when developing a case formulation in CFT:

- background experiences that bring about emotional memories and the (de)activation of affect regulation systems (e.g. harsh criticism or other forms of abuse by parents or other attachment figures; indifference or discrepant responses to emotional needs; school bullying; negative social comparison and social put-down; emotional deprivation; lack of warmth and safeness experiences; dysfunctional modelling of expectations, beliefs and behaviours);

- core fears developed within the context of the aforementioned backgrounds (e.g. fear of expressing needs, disagreement or emotions; fear of being left out or left alone; fear of being mocked or ridiculed; fear of failing or underachieving; fear that others will take advantage of us);

- safety strategies developed as means of avoiding, containing or mitigating the core fears (e.g. acting tough to hide what is perceived as weakness; using self-criticism as an attempt at selfcorrection or improvement; self-harming as an affect regulator; submissive behaviour to turn off aggression in others; threatening, to elicit fear and compliance in others; ruminating in an attempt to solve problems; suspiciousness, to anticipate threat; substance misuse to block memories and emotions; procrastinating, to postpone evaluation; isolating oneself to avoid conflicts); 
- unintended consequences (e.g. greater external control and pressure; inability to make decisions; being easily manipulated by others; disengaging from valued goals; entrapment; lethargy and fatigue; overeating and becoming overweight; increased shame and self-criticism; alienation, depersonalisation, derealisation).

Such case formulations illustrate the individual's emotional dispositions and motives. For example, children who have grown up with a lot of criticism may be overly motivated to please others in an effort to avoid criticism, and lack self-assertion and a sense of independence; they are vulnerable to feelings of anxiety, loneliness and depression. Other children may develop more aggressive defences; they are quick to anger and may threaten others if they are criticised.

\section{A focus on de-shaming}

Children and adolescents are often involuntary patients, brought to therapy by their parents, who state that there is something 'wrong' with the child, thus from the start locating the child in an external shame position. Typically, children do not fully grasp the idea of 'therapy', why they are being brought to therapy or what is expected of them in a therapeutic context (Berg 2003; Turns 2014). Given that psychotherapeutic settings involve exposure of the self to another, they are likely to elicit feelings of shame, self-contempt and personal inadequacy; therefore, these individuals are in particular need of a compassionate therapeutic engagement. The development of a 'de-shaming' rationale requires two aspects: first, to be genuinely validating of the individual's emotional experience (e.g. traumatic, adverse events are not 'cognitive distortions'); and second, to explore safety strategies and symptoms as the individual's best efforts to cope with very difficult situations (Gilbert 2014). If the rationale conveys a warm understanding of the problem (key messages include: 'we all do our best to avoid or cope with suffering' and 'this [the problem] is not your fault - it is your body's and mind's best efforts to cope and avoid being hurt in one way or another'), then children and adolescents are likely to lessen their internal shame and self-criticism, and thus feel safer and able to express emotions, and collaboratively explore more adaptive ways of coping with difficulties. Boxes 1 and 2 provide examples of developmentally appropriate deshaming therapeutic rationales (both vignettes are fictitious, though based on our clinical experience).

\section{General features of CFT}

CFT is best described as an integrated, multimodal therapy. Rather than belonging to a

\section{BOX 1 Case vignette: Jack, 8 years old, attention-deficit hyperactivity} disorder

'We all have different, unique bodies. The point is that we all have a body to take care of. If you look around you will find that some of your colleagues have pretty quiet, sometimes even floppy bodies. Some may look solid as a rock and rather stiff. Others and perhaps these will sound more familiar to you - have a hyperactive body.

'Well, there are lots of cool things about having a hyperactive body: we really enjoy the excitement of games and play, and we have lots of energy to spend - for instance, you told me that no one can beat you in running contests in the playground during recess. However, this same body is sometimes so excited and energetic that it is difficult to calm it down - especially when it gets bored. A hyperactive body needs a good "chum" - someone who is capable of fully enjoying it and of calming it down when necessary. It is just like in Formula One: you need one type of speed to run the straights and you need a different one for the curves and corners.

'My invitation is for us to meet here once a week and train our "chum" inside, so that you can overcome any type of circuit you'll have to race in your life. Then you can choose whether to be excited or calmer depending on the situation.'

single 'school' or 'model', CFT is primarily based on the research and contributions drawn from psychological sciences and is ultimately committed to enhancing the science of psychotherapy and improving the understanding and outcomes of the psychotherapeutic processes (Gilbert 2009). Therefore, CFT uses an array of established cognitive-behavioural techniques, such as Socratic questioning, exposure, functional analysis, reframing, mindfulness and chaining analysis. However, compassionate mind training (CMT) is the key therapeutic process in CFT. CMT is aimed at rebalancing the sensitivity, overactivity

\section{B0X 2 Case vignette: Rose, 15 years old, fear of bullying}

'Moving from one town to another and from one school to another is very challenging: we have to make new friends and get acquainted with what is already familiar to the others. It takes courage to face new situations and get to know new people. You told me there were times when you were bullied in your last school: a group of girls would tease you, call you names and mock you. That must have been so hurtful for you.

'Fortunately, you overcame that problem and the bullying stopped. However, we don't forget those kinds of things, and it is actually very easy to remember them and be afraid that they might happen again. As you described pretty clearly, even when others stop bullying us, we can keep on bullying ourselves. This can prevent us from living our lives the way we want to live them. For instance, you have been declining invitations to go out with some of your new colleagues who would like to know you better, or you feel it is too difficult to go to the cafeteria or the main playground during school breaks. That is completely understandable given what's happened to you before.

'The problem is if a particular situation has changed. It's a good idea to keep your umbrella up if it's raining, but maybe not when the rain stops; so sometimes we need a step-by-step plan to trust a bit. Certainly you hoped your strategy would make your life easier, but you realise that you are feeling more lonely and insecure. This is because you still want to build new friendships and have good moments of fun at school (and out of school!).

'What do you think if we could find ways of calming the "bully inside" and in the meantime letting you find the courage that has been blocked, but that you already proved you have?' 
and conflict of the threat protection and/or drive systems through the stimulation of the soothing system, which in turn provides a sense of calming, reassurance and relief that is likely to improve the patient's ability to develop emotional tolerance, generate authentic alternative thoughts and take on caring behaviours and helpful actions towards others and the self (Gilbert 2010; Leaviss 2015).

In this sense, CMT exercises (e.g. compassionate other imagery, compassionate chair work, soothing rhythm breathing, compassionate behaviour) may be regarded as part of a 'neurophysiotherapy approach' (Gilbert 2005), because patients will be training their minds in different ways: namely, in terms of experiencing safety in their interactions, tolerating distress when exploring certain themes in therapy and replacing self-criticism with self-compassion. The ultimate goal of CFT is to develop the following compassionate attributes (Gilbert 2010):

- care for well-being: motivation for self-care and prosocial behaviour, facilitating distress alleviation and flourishing

- sensitivity: the ability to be attentive to feelings, needs, suffering and distress

- sympathy: being emotionally connected, moved, engaged and attuned (as opposed to cold, distant or dissociated)

- empathy: taking the perspective of somebody else or even the perspective of a different part of ourselves (e.g. why we feel what we feel; critical self $v$. compassionate self)

- distress tolerance: the ability to contain and stay with difficult, complex or highly negative emotional states, instead of avoiding, denying or invalidating them

- non-judgement: taking an accepting, noncritical, non-condemning and de-shaming approach to emotional experiences.

These competencies help us to engage with our difficulties rather than deny, avoid or dissociate from them. In addition, there are a set of competencies that help us try to alleviate and prevent suffering or try to heal it. These include:

- how to pay attention to what is helpful

- imagining what is helpful

- using our reasoning to think through things and focus on what is helpful

- behaving in ways that address the problem even if that requires courage

- using our bodies to calm our minds

- working with our feelings.

In the CFT model, then, there are six competencies underpinning our abilities to engage with suffering, and six for working with it.

\section{Working with children and adolescents}

Children who face highly adverse and stressful situations during their development are prone to impairments in the maturation of their affect regulation systems (Gerhardt 2004). As a general therapeutic framework, the main goal of CFT is the activation of the affiliative soothing system, which will ultimately improve the effectiveness of a variety of psychological interventions (Gilbert 2009). Because the activation of the affiliative soothing system and the regulation of difficult emotions depend on care-providing relationships, the therapeutic relationship and parentchild relationships will be at the heart of CFT for children and adolescents. Cognitive-behavioural therapy has long acknowledged that the therapeutic relationship is an interpersonal context in which previous attachment problems, as well as failure in emotional validation and compassion, may be reflected, thus providing a crucial opportunity for modifying related psychological difficulties (Leahy 2008). Aligned with these claims, in CFT the qualities and behaviours displayed by the therapist and the therapist's refined view of the therapeutic relationship are an active ingredient in the change process itself. The therapist is expected to master the skills and convey the attributes of compassion, leading the patient to experience the therapeutic relationship as safe, warm, deshaming and supportive, and helping them develop a compassionate approach directed at the self and the difficulties being experienced.

People who come to therapy often display a cold, intimidating and aggressive inner tone in trying to change their thoughts and behaviours (Gilbert 2009). Children and adolescents with psychological disorders are likely to experience additional criticism within their care-providing relationships, namely from their parents (Barish 2009). Therefore, in CFT with paediatric patients, when replacing criticism, avoidance, harm and incapacitation with kindness, understanding and encouragement, a simultaneous emphasis is placed on both the young person and their interpersonal contexts (e.g. parents, teachers and peers) as possible sources of criticism and heightened psychological distress. The experience of safety, reassurance and encouragement within the therapeutic relationship is key to this process (Gilbert 2013); if this is not attained, the child or adolescent will find it difficult to generate alternative thoughts or to believe in them, to engage in new behaviours, or to validate their emotions. In CFT, logic and rational evidence are secondary to warmth. Therefore, the therapeutic qualities of motivated interest in caring, encouragement, gentleness, kindness, patience and 
a sense of wisdom are to be experienced through the therapist's corresponding behaviours, such as building on positives, looking forward, focusing on compassionate self-correction, and increasing the opportunity for engagement as well as for reparation.

\section{Compassionate mind training}

CFT argues that many psychological disorders are rooted in interpersonal problems such as difficulties in feeling cared for by others, having a caring interest in others, and having a caring attitude towards oneself. Accordingly, compassion can be experienced and developed in three directions:

- compassion we feel for others (prosocial behaviour)

- compassion we feel from others towards ourselves (e.g. the parent-child relationship)

- compassion we direct towards ourselves (selfcompassion) (Gilbert 2010).

CFT proposes that training in specific skills may be needed to develop these compassion attributes (sometimes called engagement attributes). These transformative skills include compassionate attention, compassionate reasoning, compassionate behaviour, compassionate imagery, compassionate feeling and compassionate sensation (Gilbert 2014). Although many exercises used in CMT are linked to more than one compassionate skill, the clinical techniques and exercises presented in the next sections are grouped according to the specific skill they most clearly target. Likewise, the exercises described below are selected or adapted from a wide array of techniques available in the clinical literature, in order to clearly describe the practice of CMT with children and adolescents.

\section{Building the compassionate self and image}

One of the most crucial aspects of CFT is to help individuals realise that they can choose to create a different sense of self. For example, as therapist we can imagine with the patient the core qualities we would have if we were at our most compassionate, wisest and most confident. We can imagine being that - like an actor taking on a role. Then we bring to mind a life event and imagine how we might cope with that event if we were in, for example, an anxious state of mind - what would we feel and do? Then we can move into soothing breathing and create compassionate body postures, and imagine ourselves at our compassionate best bringing wisdom, courage and commitment to the situation. How would we cope with the situation in this compassionate self position? Through this process of shifting from one mental position to another, one sense of self to another, we help our patients to realise that they can refocus how they approach difficulties. Over time, the practice of becoming the compassionate self and looking at oneself with compassion becomes part of the core therapy.

We can also use our imagination to create an image of an ideal compassionate other (a person or even an animal) that would always listen and be understanding of us, committed to being helpful. Having a patient feel that sense of compassion can itself be therapeutic, particularly if there is a high level of distrust. Again, when troubled, the patient can imagine hearing the calm soothing voice of the compassionate image. Such use of imagery can be very helpful and may enhance the effectiveness of a variety of techniques.

\section{Techniques and exercises in compassionate mind training}

\section{Compassionate attention}

The ability to pay attention to what is helpful, in a supportive way, is developed in therapy through attention training (e.g. refocusing) (Gilbert 2014). Recently, practical guidelines and exercises have been reviewed for the clinical application of mindfulness with children and adolescents (Carona 2016). Mindfulness is defined as "paying attention in a particular way: on purpose, in the present moment, and non-judgmentally' (KabatZinn 1994: p. 4). The awareness that emerges from this manner of paying attention inherently encompasses a warm, kind-hearted tone within the attending person (Kabat-Zinn 2003). Consequently, developing mindful attention skills is particularly important in CFT, because these skills enable the individual to be exposed to their painful thoughts and feelings, while embracing themselves with compassion (Neff 2015). Moreover, learning to pay attention to positive qualities and the savouring of experiences may assist the development of the patient's appreciation and gratitude (Gilbert 2009).

\section{Counting breaths}

The literature contains a considerable variety of mindful attention training exercises for young people, one of which is counting breaths. This is a very simple exercise that may be particularly helpful with younger children, who may find it difficult or boring to keep their eyes shut for longer than a moment (Ozawa de Silva 2011). The practice involves counting the breaths as a means of (re)directing attention and gaining increased awareness of the present moment. Silverton's 
(2012) detailed description is reproduced here, with a slight adaptation for the clinical context:

- ask the young person to choose a number between 3 and 10

- with their hand placed on their belly, they count on each out-breath up to the chosen number, directing their attention to breath and belly as a way of keeping the mind occupied (alternatively, or preferably the first few times, the therapist can count the breaths)

- each time they notice that their attention has wandered, they simply return to counting (once the desired number is achieved, they may want to start again).

While performing this exercise, the individual may be reminded, in a friendly and warm tone, that their breathing is always there with them, keeping them alive, helping them 'anchor' in the present moment, and taking care of them.

\section{Compassionate reasoning}

Compassionate reasoning means thinking about oneself, others and the world in ways that are helpful and supportive. In cognitive therapy, a number of techniques are implemented to achieve more balanced reasoning, such as reappraisal and reattribution. However, logic and rationality are largely insufficient for modifying one's thoughts, emotional states or behaviours; in CFT, it is crucial to ensure that the patient experiences alternative thoughts as kind, genuine and helpful (Gilbert 2009). Therefore, understanding the protective value of certain negative emotions and thoughts, and activating the affiliative soothing system through the therapeutic relationship, are necessary conditions for effectively developing compassionate reasoning skills.

\section{Compassionate chair work}

One exercise in compassionate reasoning is compassionate chair work. The patient is encouraged to change between two chairs: sitting in the first, they are invited to replicate their 'critical self'; in the second, they respond to the criticism. The exercise is kept brief, but the patient can see the impact of the criticism. Next, the therapist moves into the second chair, which 'becomes' the compassionate self that can bring wisdom, courage and commitment. It is not a good idea to encourage the compassionate self to have complex dialogues with the critical self, because this creates a focus on debate. The main focus is to help the person locate themselves in the compassionate self.

Later, the person can begin to think that the critical self lies behind a number of difficulties, as an expression of fear of rejection, thus rehearsing and training their compassionate skills and attributes (Gilbert 2010). Although this exercise may involve different levels of complexity (e.g. more than two aspects of the self), we suggest that it be kept as simple as possible when delivering it to children and adolescents.

The image of 'the bully inside our heads' may be useful in explaining the rationale for this exercise. Compassionate chair work may be particularly useful in helping patients develop a compassionate inner voice, and it may be a foremost strategy for training balanced reasoning in responding to threat-focused and self-critical thoughts. Flashcards with critical thoughts and compassionate responses may sometimes be useful in facilitating the generalisation of the insession work to real-life contexts. With younger children, the chair work may be adapted to funny role-reversal exercises in which the therapist models compassionate skills and attributes (e.g. 'Casper the Friendly Ghost', 'Shrek's Donkey'), in responding to negative remarks from the 'shy ghost' or the 'sleepy dwarf', for instance.

\section{Socratic questioning for compassionate guided discovery}

Using Socratic questioning as a means of guided discovery implies that the patient has the knowledge to answer questions that draw their attention to broader or different issues than those that are their current focus, as a process of facilitating the construction of a new idea or a different appraisal (Padesky 1993). This technique is generally suitable for older adolescents, who have evolved the cognitive abilities for abstract thinking, and it can be used to lead them to gain insight into the importance and the possibility of introducing a generous quality into coping with difficulties. The following questions may assist in achieving that purpose:

- 'If, by magic, you could stop criticising yourself, what would be your biggest fear?'

- 'What would be so bad about that?'

Usually, a self-corrective aim comes out as an answer. For example, the young person may say: 'If I wasn't self-critical, I wouldn't work so hard, I wouldn't reach my potential; maybe I would make mistakes or maybe not bother to avoid hurting others'. Here, as therapist we acknowledge these good goals, but wonder whether self-criticism is the way to achieve them. We might invite the young person to do a behavioural experiment - to 'meet' the critical self. We might ask:

- 'Can you give me an example of something you don't like about yourself and that you criticise 
yourself for? Can you give me an example related to the reason you're here?'

- 'Would you be willing to let go of this internal critic - this bully that keeps kicking you when you are already on the floor?'

We then invite the individual to begin to explore the critical self. First, we spend a few moments imagining the critical self; what would it look like if you could take it out of your head and look at it? Next, we pay attention to what is it is actually saying, allowing ourselves to really hear its words; then we pay attention to the feelings it arouses in us. Finally, we note how we feel when we do this. We can then explore with the patient their experience. Typically, the critical self will look hostile in some way, it will say unpleasant things, it will direct unpleasant emotions (anger, contempt) towards the self. We can explore the question 'Does this critical self help you achieve your self-corrective aim?', i.e. we examine the unhelpfulness of self-criticism as a way of selfcorrection and the patient's reluctance to let go of this ineffective behaviour.

Individuals quickly see that their self-criticism is actually a lot more damaging than they realised, and a lot more unpleasant. In CFT we very rarely try to argue with the critical self, but rather help the individual to refocus on the compassionate self. Keeping the same goals, we go into the breathing and focus of the compassionate self - allowing it to settle into the body - then asking 'What does your compassionate self or image look like?'; 'What is it saying to you?'; 'What does it feel for you?'; and, finally, 'How are you feeling now?'

The aim is that the patients themselves begin to have an experience of contrasting hostile selfcriticism with compassionate self-correction and encouragement. Ultimately, they will see the value in the compassionate focus.

\section{Compassionate behaviour}

Compassionate behaviour may involve doing things that are helpful for the self (e.g. exposing oneself to feared stimuli, painful experiences, avoided situations) or for others (e.g. offering help if someone is hurt or upset). The core aspect of compassionate behaviour training is the validation and development of the patient's courage, particularly through modelling the acknowledgement of any constructive effort in confronting their difficulties (Gilbert 2009, 2013). Traditionally, the (graded) behavioural task assignment is the major therapeutic method in training compassionate behaviour, and invariably involves exposure to both negative and positive emotions. Nevertheless, a number of other strategies may be valuable in facilitating the development of compassionate behavioural patterns. In one such strategy, prosocial behaviour is directly targeted as a way of translating compassionate behaviour derived from the self to others (Gilbert 2010).

\section{Fostering prosocial behaviour}

Prosocial behaviour refers to 'voluntary actions that are intended to help or benefit another individual or group of individuals' (Eisenberg 1989: p. 3). It includes positive interactions such as helping, sharing, cooperating and comforting (Hay 1994). Current research suggests that happiness, popularity and prosociality are not only compatible goals, but also reciprocal (Layous 2012); in fact, there is increasing evidence for the assertion that 'it's good to be good', i.e. for the role of prosocial behaviour as a determinant of positive health outcomes (Post 2014). Additionally, it has been noted that prosocial behaviour often leads to the experience of a 'warm glow' and that its emotional benefits may be observable even in very young children (Aknin 2012).

In CFT with children and adolescents, behaviours such as caregiving, teaching, assisting, protecting and soothing are to be carefully monitored and positively reinforced across all life contexts (e.g. home, school, leisure activities). Tasks demonstrating empathy, kindness and concern for the needs of others (e.g. allowing a sibling to take the first turn in a video game, standing up for someone who is being bullied, helping with a school fundraiser) are often assigned as part of psychological intervention protocols for children with behavioural disorders (Jongsma 2006). Nevertheless, it is worth highlighting that in the context of a compassionate therapeutic relationship and process, prosocial behaviours should be shaped and modelled rather than merely taught or assigned.

\section{Compassionate feeling}

Compassionate feeling encompasses the experience of kindness and affiliation (compassion for others and from others), as well as the ability to understand negative emotional states (e.g. anger, anxiety, shame) and turn them into self-compassionate behaviour (e.g. assertiveness). Validating the young person's emotional experiences is a continuous (rather than a discrete) therapeutic strategy aimed at de-shaming, and is therefore at the heart of CFT. Therapeutic validation may be defined as the radical acceptance and acknowledgement that all behaviour has validity and is understandable, and it is particularly crucial when working with young people from invalidating environments (Salsman 2012). 
Developing the young person's emotional philosophy

Validation implies finding truth in what one feels and thinks, and it is therefore intermediate between empathy and compassion (Leahy 2005). When working with children and adolescents, the therapist should be mindful of the young person's pathological styles of validation (e.g. 'If you cared about me, you would agree with everything I say'; 'If you cared about me, you wouldn't try to change the way I feel') and self-invalidating strategies (e.g. 'Needing others means I am weak'; 'There's no point in talking about things I can't change'). Within this social-cognitive model of validation (Leahy 2005), two strategies may help the individual in developing their 'emotional philosophy' (i.e. a compassionate understanding of one's emotional states and experiences): first, to emphasise the importance of all emotions (not just 'feeling good', as illustrated in the animated film 'Inside Out'); second, to teach how emotions contain one's meanings and needs. In this context, unrealistic expectations and beliefs about emotional well-being and regulation (e.g. 'to be happy is to be always cool', 'it is ridiculous to get nervous in such situations', 'losing my temper means I am a bad person') are to be challenged and readily addressed as emotional or mind traps, given their counterproductive consequences. When exploring the nature and diversity of human emotional experience, the therapist may facilitate the young person's insight into how negative emotional states are (or may be) linked to positive outcomes through one's helpful behaviour (e.g. regret makes us apologise and/or correct the consequences of our unintended actions; anxiety reminds us about our goals and gives us a boost to act on them; anger is perfectly understandable and greatly useful when standing up for injustices).

With younger children or with adolescents who have severely impaired emotional development, it will often be necessary to work on their emotional vocabulary and on the recognition of feelings in themselves and in others (Joseph 2003). Currently, a number of clinical resources (including activities, books and games) are easily accessible for this purpose - for example, see the Center on the Social and Emotional Foundations for Early Learning (http://csefel.vanderbilt.edu/).

\section{Compassionate imagery}

Given their physiological power, imagery exercises (along with meditation-like practices) are greatly valued in CFT as a means of stimulating the affiliative soothing system (Gilbert 2014). Exercises that directly target imagery in CMT include safe place imagery (generating an image of a place where the individual feels safe and soothed); compassionate colour imagery (imagining a colour that the young person associates with warmth and kindness and imagining it having a motivation to help); ideal compassionate other imagery (creating an ideal image of a compassionate other who has three key qualities: wisdom, strength, and commitment to care); and compassionate self imagery (imagining the self who has the same three qualities: wisdom, strength, and commitment to care) (Gilbert 2010).

\section{Loving kindness meditation for children}

There are different techniques for loving kindness mediation (Hoffmann 2011), but an ageappropriate adaptation for children has been developed by Saltzman \& Goldin (2008). The procedure for this loving kindness practice is as follows.

- Ask the child to remember a time they felt loved by someone (it can be a very simple moment with a parent, grandparent, teacher, friend or pet).

- Invite the child to really feel this loving moment and feel the love flowing between themselves and the person or animal who loves them (younger children may enjoy blowing kisses, whereas older children can simply imagine receiving and sending love in and from their hearts).

- The child can experiment with sending love to someone they do not know very well (e.g. a server in the school cafeteria, a bus driver).

- The child might then think about someone they are having troubles with, such as their 'ex-best friend'.

- The exercise can be finished with the child's thoughts of sending love to themselves and feeling their love returning to them, and then sending love to the whole world and feeling the whole world's love returning to them.

\section{Compassionate sensation}

In CFT, sensory work includes breathing exercises, tones of voice and facial/body postures to generate physical sensations (e.g. activation of the parasympathetic system), resulting in affect regulation and compassion - which ultimately involves exposure to difficult internal and external stimuli. In addition to mindfulness, soothing rhythm breathing is a preferred technique in CMT for achieving this type of compassionate sensation (Gilbert 2010).

\section{Soothing rhythm breathing}

The easiest way to familiarise children and adolescents with soothing rhythm breathing is 
to teach them to pause for nearly three seconds between the in-breath and the out-breath - this moment of pause has been labelled 'the still quiet place' in a mindfulness programme for children and adolescents (Saltzman 2014). If repeated at least three times, the pauses between breaths allow the activation of the parasympathetic system, resulting in the person feeling more relaxed and grounded. Another way of teaching soothing rhythm breathing to children and adolescents is counting from 1 to 10 on the exhale (Greenland 2010): this technique simply involves relaxing as one inhales and counting from 1 to 10 as one exhales (usually, one has to count pretty quickly). Another possibility is to teach gradual soothing breathing, beginning with counting $1-2-3$ on the in-breath, and (following a brief pause of up to 3 seconds) $1-2-3$ on the out-breath; the number can then be gradually increased (between 5 and 7 would be a reasonable goal for most children and adolescents).

\section{Developmental considerations: the importance of a parent-child approach}

Generally, parents are the primary attachment figures for a child. Healthy attachment relationships ensure that children seek proximity to attachment figures, have a sense of protection and being soothed, and develop a secure base from which to explore the world. During early life, the development of basic circuits of the brain - such as those responsible for the mental processes of emotion generation and regulation; the capacity to adopt flexible, mindful and reflective behaviour; the autobiographical sense of self; and the ability to engage in interpersonal communication and to experience empathy - is largely shaped by the quality of the child's interpersonal experiences. There are five core elements to foster in caregiving attachment relationships (Siegel 2001):

- collaboration and non-verbal communication (eye contact, facial expression, synchrony of responses; a soothing/encouraging tone of voice; the experience of 'feeling felt' by others)

- reflective dialogue (creating meaning for experiences; creating the representations of the mind of others and of the self)

- repair (allowing oneself to make sense of periods of painful disconnection and psychological distress)

- coherent narratives (creating an autobiographical narrative and self-awareness)

- emotional communication (attachment relationships can amplify the positive affect experienced in joyful moments of living, as well as reducing negative emotional states through sharing, validation and soothing).
Therefore, targeting parent-child relationships and interactions becomes of paramount importance in CFT with children and adolescents. The idea of engaging parents as 'proto-therapists' in their child's treatment is emphasised by the fact that parents provide the most permanent context for the development of their child's maturing brain. In fact, psychotherapy may be defined as the encounter of (at least) two minds with the shared aim of lessening psychological suffering (Gilbert 2014). To this end, the idea of 'mind' can be put in terms of patterns in the flow of energy and information, which can occur within one brain or between brains (Siegel 2001). Accordingly, psychotherapy is concerned with the repair of impediments to flexible self-regulation and coherent self-knowledge that often result from blockage or other impairments in the flow of energy and information within the mind and between minds (Siegel 2001). In other words, CFT with children and adolescents should be primarily aimed at fostering compassionate parenting behaviours, as briefly illustrated by the following therapeutic exercises and techniques.

\section{Replacing (self-)criticism with compassionate (self-)correction}

Parents often 'mis-take' criticism for correction, towards either themselves or their child. This perception is largely due to what has been termed 'fundamental educational error' in developmental psychology: the belief that one's 'bad behaviour' (e.g. failures or mistakes) demands punishment, whereas one's 'good behaviour' calls for no particular consequence, because it was the expected, adequate or desirable (Lourenço 1992). Such beliefs impede parents from noticing and encouraging their child's or their own efforts to manage difficulties, and they eventually foster critical, unhelpful behaviours. This phenomenon can be illustrated by the clinical observation that parents often bring their child to therapy and present their difficulties with a critical tone, while blaming themselves and becoming easily selfcritical about their parenting efforts.

Through Socratic questioning, parents may be invited to reflect on the perceived advantages of criticism and its actual outcomes, its links to their own attachment experiences as children, and its association with mindless parenting behaviours. Within this guided discovery, parents may be asked to imagine that they have to choose one of two schools for their child (or for themselves). In the first school, children are under constant pressure to 'do it right', and therefore their slight mistakes are immediately pointed out and criticised by 
their teachers, who want them to attain their best academic performance. In the second school, there is also a strong commitment to positive outcomes, but every time a child fails, they are reassured by teachers and given the opportunity to face and manage difficulties, and to correct the work or behaviour. The parents are then asked: bearing your child's best interests in mind, which school would you choose? This simple metaphor (Gilbert 2009) may well serve as a starting point for parents to question the nature, value and outcomes of their critical approach to difficulties.

\section{Loving kindness meditation}

Mindful parenting involves the ability to experience compassion for the self and the child (Duncan 2009). As in typical CMT, parents are encouraged to practise exercises that are likely to foster the acknowledgement of a common humanity and the development of compassionate skills and attributes towards themselves and their children. This practice may be recommended for parents of typically developing children, or parents of children presenting internalising or externalising problems, but it may be particularly important for parents of children with developmental disabilities. In these cases, CMT in general, and loving kindness meditation in particular, may assist to counteract the maladaptive cycle of shame (internalised stigma), guilt and self-blame in which these parents frequently find themselves trapped (Neff 2014).

Box 3 presents an adapted version of the loving kindness (metta) meditation proposed by Shapiro $\&$ White (2014) for the context of parenting.

\section{Conclusions}

CFT provides a multimodal therapy framework that can enhance the effectiveness of other psychological interventions. There is now consistent evidence for a negative association between self-compassion and psychopathology (MacBeth 2012), as well as preliminary evidence for the clinical effectiveness of CFT, particularly for people with high self-criticism (Leaviss 2015). The practice of compassionate loving kindness meditation has been proved to be effective in activating the neural circuitries linked to empathy (Lutz 2008), as well as in reducing negative mood and increasing feelings of social connection, positive emotions and mindfulness (Hutcherson 2008). Likewise, self-compassion was found to be linked to the emotional well-being of pre-adolescents and adolescents (Bluth 2014, 2015), and the effectiveness of a cognitive-based compassion-training programme for the reduction

\section{B0X 3 Loving kindness meditation for parents}

The parents are invited to sit comfortably and to allow themselves to connect with their bodies and breathing (e.g. by placing a hand over the heart and feeling the warmth sensations).

They are encouraged to invite an intention to this practice (e.g. 'to open my heart', 'to invite compassion into my life', 'to give kindness a moment').

They recite to themselves: 'May I be safe. May I be happy. May I be healthy. May I live with ease. May I be free from suffering', or, alternatively, 'May I find the courage, the strength and the wisdom to help me out in this situation'.

If they become aware of any resistance to accepting these wishes for themselves (e.g. intrusive images, evaluative thoughts), they should simply notice those thoughts and/or feelings and gently return to the practice of reciting the phrases.

For the next part of the practice, the parents are asked to call their child to mind (e.g. bringing different images of the child to mind or just recalling a specific moment). They repeat the same loving kindness phrases directed to the child, while noticing and feeling the compassionate sensations: 'May you be safe. May you be happy. May you be healthy. May you live with ease. May you be free from suffering'

(After Shapiro \& White, 2014)

of inflammatory markers (which are believed to predict the risk for medical or psychiatric illnesses) has been reported in a study conducted with adolescents living in foster care (Pace 2013). Nevertheless, given the relative nascence of CFT as a formal psychotherapeutic model and the considerable complexity of its conceptual and clinical framework, in-depth clinical training is required to ensure the clinician's mastery and effectiveness in delivering CFT with younger populations.

New developments concerning the applications of CFT for children and adolescents are likely to arise in the coming years. These developments will hopefully expand the current evidence base for what appears to be a solid and promising model of psychotherapy.

\section{Funding}

C.C., D.R., C.S. and P.C. are supported by the Cognitive and Behavioral Center for Research and Intervention (Faculty of Psychology and Education Sciences, University of Coimbra). C.C. is also supported by the Cerebral Palsy Association of Coimbra. 


\section{Acknowledgements}

The authors thank Fernanda Maurício for compiling a number of clinical resources, and Diana Ribeiro da Silva for her help with the organisation of references.

\section{References}

Addis ME, Carpenter KM (2000) The treatment rationale in cognitive-behavioral therapy: psychological mechanisms and clinical guidelines. Cognitive and Behavioral Practice, 7: 147-56.

Aknin LB, Hamlin JK, Dunn EW (2012) Giving leads to happiness in young children. PLOS ONE, 7: e39211.

Baldwin DA (1997) The concept of security. Review of International Studies, 23: 5-26.

Barish K (2009) Emotions in Child Psychotherapy: An Integrative Framework. Oxford University Press.

Berg I, Steiner T (2003) Children's Solution Work. W. W. Norton \& Company.

Bluth K, Blanton PW (2014) Mindfulness and self-compassion: exploring pathways to adolescent emotional well-being. Journal of Child and Family Studies, 23: 1298-309

Bluth K, Blanton PW (2015) The influence of self-compassion on emotional well-being among early and older adolescent males and females. Journal of Positive Psychology, 10: 219-30.

Bowlby J (1980) Loss: Sadness and Depression. Vol. 3: Attachment and Loss. Hogarth Press.

Brown SL, Brown RM (2015) Connecting prosocial behavior to improved physical health: contributions from the neurobiology of parenting. Neuroscience \& Biobehavioral Reviews, 55: 1-17.

Carona C, Moreira H, Silva N (2016) Therapeutic applications of mindfulness in paediatric settings. Advances in Psychiatric Treatment, 22: 16-24.

Castilho P, Pinto-Gouveia J, Amaral V, et al (2014) Recall of threat and submissiveness in childhood and psychopathology: the mediator effect of self-criticism. Clinical Psychology and Psychotherapy, 21: 73-81.

Castilho P, Pinto-Gouveia J, Duarte J (2015) Exploring self-criticism: confirmatory factor analysis of the FSCRS in clinical and nonclinical samples. Clinical Psychology and Psychotherapy, 22: 153-64.

Cozolino L (2007) The Neuroscience of Human Relationships: Attachment and the Developing Brain. Norton.

Crocker J, Canevello A (2012) Consequences of self-image and compassionate goals. In Advances in Experimental Social Psychology (eds P Devine, A. Plant): 229-77. Academic Press.

Depue RA, Morrone-Strupinsky JV (2005) A neurobehavioral model of affiliative bonding. Behavioral and Brain Sciences, 28: 313-95.

Duncan LG, Coatsworth JD, Greenberg MT (2009) A model of mindful parenting: implications for parent-child relationships and prevention research. Clinical Child and Family Psychology Review, 12: 255-70.

Eisenberg N, Mussen PH (1989) The Roots of Prosocial Behavior in Children. Cambridge University Press.

Fredrickson BL, Grewen KM, Coffey KA, et al (2013) A functional genomic perspective on human well-being. Proceedings of the National Academy of Sciences, 110: 13684-9.

Gerhardt S (2004) Why Love Matters: How Affection Shapes a Baby's Brain. Brunner-Routledge.

Gilbert P, Irons C (2005) Focused therapies and compassionate mind training for shame and self-attacking. In Compassion: Conceptualizations, Research and Use in Psychotherapy (ed P Gilbert): 263-325. Routledge.

Gilbert P (2007) The evolution of shame as a marker for relationship security. In The Self-Conscious Emotions: Theory and Research (eds JL Tracy, RW Robins, JP Tangney): 283-309. Guilford Press.
Gilbert P, McEwan K, Mitra R, et al (2008a) Feeling safe and content: a specific affect regulation system? Relationship to depression, anxiety, stress and self-criticism. Journal of Positive Psychology, 3: 182-91.

Gilbert P, Irons C (2008b) Shame, self-criticism, and self-compassion in adolescence. In Adolescent Emotional Development and the Emergence of Depressive Disorders (eds NB Allen, LB Sheeber): 195-214. Cambridge University Press.

Gilbert $\mathrm{P}$ (2009) Introducing compassion-focused therapy. BJPsych Advances, 15: 199-208.

Gilbert P (2010) Compassion Focused Therapy. Distinctive Features. Routledge.

Gilbert P (2013) The Compassionate Mind. Constable Robinson.

Gilbert $P(2014)$ The origins and nature of compassion focused therapy. British Journal of Clinical Psychology, 53: 6-41.

Gold J, Sullivan MW, Lewis M (2011) The relation between abuse and violent delinquency: the conversion of shame to blame in juvenile offenders. Child Abuse and Neglect, 35: 459-67.

Greenland SK (2010) The Mindful Child: How to Help Your Kid Manage Stress and Become Happier, Kinder, and More Compassionate. Free Press.

Hay DF (1994) Prosocial development. Journal of Child Psychology and Psychiatry, 33: 29-71.

Hoffmann SG, Grossman P, Hinton DE (2011) Loving-kindness and compassion meditation: potential for psychological intervention. Clinical Psychology Review, 13: 1126-32.

Hutcherson CA, Seppala EM, Gross JJ (2008) Loving-kindness meditation increases social connectedness. Emotion, 8: 720-4.

Jongsma AE, Peterson LM, McInnis WP, et al (2006) The Child Psychotherapy Treatment Planner. John Wiley \& Sons

Joseph GE, Strain PS (2003) Enhancing emotional vocabulary in young children. Young Exceptional Children, 6: 18-26.

Kabat-Zinn J (1994) Wherever You Go, There You Are: Mindfulness Meditation in Everyday Life. Hyperion.

Kabat-Zinn J (2003) Mindfulness-based interventions in context: past, present, and future. Clinical Psychology: Science and Practice, 10: $144-56$

Keltner D, Kogan A, Piff PK, et al (2014) The sociocultural appraisals, values, and emotions (SAVE) framework of prosociality: core processes from gene to meme. Annual Review of Psychology, 65: 425-60.

Kirby JN (2016) Compassion interventions: the programmes, the evidence, and implications for research and practice. Psychology \& Psychotherapy, doi: 10.1111/papt.12104

Klimecki OM, Leiberg S, Ricard M, et al (2014) Differential pattern of functional brain plasticity after compassion and empathy training. Social Cognitive and Affective Neuroscience, 9: 873-9.

Layous K, Nelson SK, Oberle E, et al (2012) Kindness counts: prompting prosocial behavior in preadolescents boosts peer acceptance and wellbeing. PLOS ONE, 7: e51380.

Leahy RL (2005) A social-cognitive model of validation. In Compassion: Conceptualizations, Research and Use in Psychotherapy (ed P Gilbert): 195-217. Brunner-Routledge.

Leahy RL (2008) The therapeutic relationship in cognitive-behavioral therapy. Behavioral and Cognitive Psychotherapy, 36: 769-77.

Leaviss J, Uttley L (2015) Psychotherapeutic benefits of compassionfocused therapy: an early systematic review. Psychological Medicine, 45: 927-45.

LeDoux J (2003) The emotional brain, fear and the amygdala. Cellular and Molecular Neurobiology, 23: 727-38.

Lourenço 0 (1992) Erro educacional fundamental: teoria, dados e implicações [Fundamental educational error: theory, data and implications]. Psicologia, 8: 385-92.

Lutz A, Brefczynski-Lewis J, Johnstone T, et al (2008) Regulation of the neural circuitry of emotion by compassion meditation: effects of meditative expertise. PLOS ONE, 3: e1897.
MCO answers

1 b 2 c 3 e 4 c 5 e 
MacBeth A, Gumley A (2012) Compassion and mental health: a meta-analysis of the association between self-compassion and psychopathology. Clinical Psychology Review. 32: 545-52.

Matos M, Pinto-Gouveia J, Gilbert P (2013) The effect of shame and shame memories on paranoid ideation and social anxiety. Clinical Psychology and Psychotherapy, 20: 334-49.

Mikulincer M, Shaver PR (2007) Attachment in Adulthood: Structure, Dynamics, and Change. Guilford Press.

Neff KD, Faso DJ (2014) Self-compassion and well-being in parents of children with autism. Mindfulness, 6: 938-47.

Neff KD, Dahm KA (2015) Self-compassion: what it is, what it does, and how it relates to mindfulness. In Handbook of Mindfulness and SelfRegulation (eds BD Ostafin, MD Robinson, BP Meier): 121-37. Springer.

Ozawa de Silva B, Dodson-Lavelle B (2011) An education of heart and mind: practical and theoretical issues in teaching cognitive-based compassion training to children. Practical Matters, 1: 1-28.

Pace TWW, Negi LT, Dodson-Lavelle B, et al (2013) Engagement with cognitively-based compassion training is associated with reduced salivary C-reactive protein from before to after training in foster care program adolescents. Psychoneuroendocrinology. 38: 294-9.

Padesky C (1993) Socratic Questioning: Changing Minds or Guided Discovery? Keynote address to the European Congress of Behavioural and Cognitive Therapies, London, 24 September 1993

Perry BD, Pollard RA, Blakley TL, et al (1995) Childhood trauma, the neurobiology of adaptation, and "use-dependent" development of the brain: how "states" become "traits". Infant Mental Health Journal, 16: 271-91.

Post SG (2014) It's Good To Be Good: 2014 biennial scientific report on health, happiness, longevity, and helping others (http://www. stephengpost.com/downloads/Good\%20to\%20Be\%20Good\%20VI.pdf|.
Puglia MH, Lillard TS, Morris JP, et al (2015) Epigenetic modification of the oxytocin receptor gene influences the perception of anger and fear in the human brain. Proceedings of the National Academy of Sciences, 112: 3308-13.

Ribeiro da Silva D, Rijo D, Salekin RT (2015) The evolutionary roots of psychopathy. Aggression and Violent Behavior, 21: 85-96.

Salsman NL, Arthur R (2012) Adapting dialectical behavior therapy to help suicidal adolescents. Current Psychiatry, 10: 18-23.

Saltzman A, Goldin P (2008) Mindfulness-based stress reduction for school-age children. In Acceptance and Mindfulness Treatments for Children and Adolescents: A Practitioner's Guide (eds SC Hayes, LA Greco): 139-61. New Harbinger Publications.

Saltzman A (2014) A Still Quiet Place: A Mindfulness Program for Teaching Children and Adolescents to Ease Stress and Difficult Emotions. New Harbinger Publications.

Schore AN (1994) Affect Regulation and the Origin of the Self: The Neurobiology of Emotional Development. Erlbaum.

Shapiro SL, White C (2014) Mindful Discipline. New Harbinger.

Siegel DJ (2001) Toward an interpersonal neurobiology of the developing mind: Attachment relationships, "mindsight," and neural integration. Journal of Infant Mental Health, 22: 67-94.

Silverton S (2012) The Mindfulness Breakthrough. Watkins Publishing.

Tangney JT, Tracy JL (2012) Self-conscious emotions. In Handbook of Self and Identity (eds MR Leary, JP Tangney): 446-80. Guilford Press.

Turns BA, Kimmes J (2014) I'm NOT the problem! Externalizing children's "problems" using play therapy and developmental considerations. Contemporary Family Therapy, 36: 135-47.

\section{MCOs}

Select the single best option for each question stem

1 In compassion-focused therapy (CFT), regulation of the threat defence and/or drive systems is best achieved through:

a suppression of the over-activated system(s)

b stimulation of the soothing system

c rational dispute of maladaptive beliefs

d facilitation of corrective self-criticism

e logical analysis of the pros and cons of maladaptive behaviours.

\section{Case formulation in CFT is best described as} the articulation between:

a early maladaptive schemas, precipitating factors and avoidance patterns

b past and current object relations

c background experiences, core fears, safety strategies and unintended consequences d childhood traumas, dysfunctional selfregulation and self-criticism

e lack of compassion, fear of compassion and compassion avoidance.

3 In compassionate mind training (CMT), transformative skills do not include:

a compassionate reasoning

b compassionate behaviour

c compassionate attention

d compassionate imagery

e compassionate self-attack.

4 In CFT with children and adolescents, CMT exercises:

a can only be performed with older adolescents

b are exclusively focused on parent-child relationships

c can be tailored to the patient's age

$\mathrm{d}$ are indicated for internalising problems only

e are all based on meditation practices.
5 A parent-child perspective in CFT means:

a keeping coercive parents away from therapy

b reinforcing parents' self-criticism to modify inadequate parenting

c always having parents and their children together in therapy sessions

$\mathrm{d}$ helping parents distance themselves from their child's problems

e assisting the development of compassionate parenting behaviour. 